\title{
Impact of Pneumococcal Conjugate Vaccine on Pediatric Tympanostomy Tube Insertion in Partial Immunized Population
}

\author{
Mao-Che Wang, ${ }^{1,2,3}$ Ying-Piao Wang, ${ }^{3,4,5}$ Chia-Huei Chu, ${ }^{1,2}$ Tzong-Yang $\mathrm{Tu}^{1,2}$ \\ An-Suey Shiao, ${ }^{1,2}$ and Pesus Chou ${ }^{3}$ \\ ${ }^{1}$ Department of Otolaryngology Head and Neck Surgery, Taipei Veterans General Hospital, Taipei, Taiwan \\ ${ }^{2}$ School of Medicine, National Yang-Ming University, Taipei, Taiwan \\ ${ }^{3}$ Institute of Public Health and Community Medicine Research Center, National Yang-Ming University, Taipei, Taiwan \\ ${ }^{4}$ Department of Otolaryngology Head and Neck Surgery, Mackay Memorial Hospital, Taipei, Taiwan \\ ${ }^{5}$ Department of Audiology and Speech Language Pathology and School of Medicine, Mackay Medical College, New Taipei City, Taiwan
}

Correspondence should be addressed to Pesus Chou; pschou@ym.edu.tw

Received 11 December 2014; Accepted 26 February 2015

Academic Editor: Eduardo G. Pérez-Yarza

Copyright (c) 2015 Mao-Che Wang et al. This is an open access article distributed under the Creative Commons Attribution License, which permits unrestricted use, distribution, and reproduction in any medium, provided the original work is properly cited.

\begin{abstract}
Objective. To investigate the impact of seven-valent pneumococcal conjugate vaccine on tube insertions in a partial immunized pediatric population. Study Design. Retrospective ecological study. Methods. This study used Taiwan National Health Insurance Research Database for the period 2000-2009. Every child under 17 years old who received tubes during this 10-year period was identified and analyzed. The tube insertion rates in different age groups and the risk to receive tubes in different birth cohorts before and after the release of the vaccine in 2005 were compared. Results. The tube insertion rates for children under 17 years of age ranged from 21.6 to 31.9 for 100,000 persons/year. The tube insertion rate of children under 2 years old decreased significantly after 2005 in period effect analysis ( $\beta=-0.074, P<0.05$, and the negative $\beta$ value means a downward trend) and increased in children 2 to 9 years old throughout the study period (positive $\beta$ values which mean upward trends, $P<0.05$ ). The rate of tube insertion was lower in 2004-2005 and 2006-2007 birth cohorts than that of 2002-2003 birth cohort (RR $=0.90$ and $0.21,95 \%$ CI $0.83-0.97$ and 0.19-0.23, resp.). Conclusion. The seven-valent pneumococcal conjugate vaccine may reduce the risk of tube insertion for children of later birth cohorts. The vaccine may have the protective effect on tube insertions in a partial immunized pediatric population.
\end{abstract}

\section{Introduction}

Acute otitis media (AOM) is a very common otologic problem in children. Sixty percent of infants experience AOM by the age of 18 months [1]. AOM usually comes after a viral upper respiratory tract infection. Symptoms and signs are the acute onset of otalgia, fever, hearing deterioration, nausea, vomiting, and otorrhea, if tympanic membrane ruptures. Streptococcus pneumoniae is the most frequently identified pathogen, which accounts for 25 to $50 \%$ of AOM, followed by Haemophilus influenzae and Moraxella catarrhalis, which are responsible of 15 to $30 \%$ and 3 to $20 \%$ of AOM episodes, respectively [2]. AOM can resolve spontaneously or after treatment. The condition can also develop into otitis media with effusion (OME) or recurrent AOM. Ninety percent of children have an episode of OME before school age, especially from the age of 6 months to 4 years $[3,4]$. Most OME resolves within three months, but $30-40 \%$ of patients may have recurrent $\mathrm{OME}[3,5,6]$. Observation and medical treatment are conservative management strategies for AOM and OME. Tympanostomy tube insertion is the preferred surgical procedure for these conditions. The American Academy of Otolaryngology-Head and Neck Surgery (AAO-HNS) set the clinical practice guidelines for OME in 2004 and clinical practice guidelines for tympanostomy tubes in children in 2013. The guidelines recommended that clinicians offer bilateral tympanostomy tubes to children with bilateral chronic OME (OME lasting for 3 months or longer) and recurrent AOM with middle ear effusion. The guidelines also recommended that clinicians should not perform tympanostomy tubes to 
children with a single episode of OME lasting less than 3 months or recurrent AOM without middle ear effusion [7, 8]. Tympanostomy tube insertion is the treatment of choice for pediatric recurrent acute otitis media and chronic otitis media with effusion.

The seven-valent pneumococcal conjugate vaccine (PCV7) was released in the United States in the year 2000. This vaccine was used to prevent pediatric pneumococcal disease. After immunization with PCV7 had been ongoing for more than a decade all over the world, it was demonstrated to be very effective in reducing mortality related to pneumococcal disease, invasive pneumococcal disease (IPD), pneumonia, and otitis media in children [9-15]. Immunization with PCV7 also reduced penicillin nonsusceptible strains of pneumococcus and vaccine type pneumococcus colonization in the nasopharynx. Serotype changes in IPD pathogens and nasopharyngeal carriage were also noted [16-27]. The protective effect against pneumococcal disease of PCV7 vaccination was found not only for vaccinated children, but also for unvaccinated children and adults. Many studies found that IPD and pneumonia of adults decreased significantly after increased coverage rate of PCV7 vaccination in infants and children, which showed there were both direct and indirect (herd) effects of the vaccine $[10,17,28-35]$. In addition to protective effects against pneumonia and IPD, PCV7 also reduced pediatric otitis media and the rate of tympanostomy tube insertions. In comparison with pneumonia and IPD, otitis media affects more children and it is associated with a greater social and economic burden than any other pneumococcal diseases [36-38]. During the past decades, numerous studies have tried to identify the protective effect of PCV7 on pediatric otitis media. Some studies were randomized controlled trials [39-46], some were analyses of secondary data [9, 47-53], and others were systemic reviews [11, 54-59]. Most studies showed PCV7 did have the protective effect on pediatric otitis media in the perspectives of reducing AOM episodes, AOM visits, physician claims for otitis media, antibiotic prescriptions, and tympanostomy tube insertion rates $[9,11,39-53,57-59]$. However, some studies of the vaccine showed negative results [54-56]. These studies all focused on children vaccinated with PCV7.

PCV7 was first introduced to Taiwan in the year 2005. It was not included into the national immunization program and was only free for high-risk children such as children with rare disease or malignancy, or parents had to pay USD 110 for each dose of the vaccine from their own pocket. We conducted this ecological study to examine the impact of PCV7 on reducing the rate of pediatric tympanostomy tube insertions, which was a surrogate of complicated and intractable pediatric otitis media in a partial immunized pediatric population.

\section{Materials and Methods}

This ten-year study (2000-2009) used Taiwan National Health Insurance Research Database (NHIRD), populationbased data on approximately $99 \%$ of the 23 million legal residents covered by the National Health Insurance in Taiwan.
Every admission and outpatient visit record was included in this database. National Health Insurance is the only buyer of medical service in Taiwan and NHIRD is released for academic use yearly by the National Health Institute of Taiwan. This study was reviewed and approved by the institutional review board of the Taipei Veterans General Hospital. All children who had tympanostomy tube insertion before they reached 17 years of age (2000 to 2009) were included in this study. The study population was obtained by retrieving all of the patients with the procedure codes for myringotomy with ventilation tube insertion under a microscope, from the claims data of the NHIRD, with ages under 17 years on the date of the surgery. This was a population-based data without any sampling. We stratified the children into four age groups $(0 \leqq$ age $<2,2 \leqq$ age $<5,5 \leqq$ age $<9$, and $9 \leqq$ age $<17$ ). We examined the rate of tympanostomy tube insertion in each age group in each calendar year during the study period from 2000 to 2009 . We also compared the rate of tympanostomy tube insertion of children in 2002-2003, 2004-2005, and 2006-2007 birth cohorts.

\section{Statistical Analysis}

Age and calendar year-specific rates of pediatric tympanostomy tube insertion were calculated as the number of children who had undergone tympanostomy tube insertion divided by the total registered population per 100000 in the given stratum of age and year. The registered population was obtained from the Taiwan National Statistics Report [60]. A graphical approach was used to describe the age-specific rates of tympanostomy tube insertion over calendar years and to describe the rates across ages by birth cohorts.

We used the Poisson regression method to examine time trend of rates of tympanostomy tube insertion. Given that pneumococcal conjugate vaccine was introduced and has been available since 2005 in Taiwan, we assumed that the period effects could have varied before and after 2005. As a result, a segmented regression analysis, also known as piecewise regression, was used to identify intervention effects of vaccine being introduced in Taiwan on the rates of tympanostomy tube insertion [61]. Period effects were separated into two segments, one for trend before 2005 and the other after 2005, and thus the time trend of rates of tympanostomy tube insertion can be estimated by two regression parameters.

A multiphase method was used to examine possible cohort effects [62]. This approach considers cohort effects as the systemic component of multiplicative interaction between age and calendar year. The first step is to examine whether cohort effect exists by using graph of age-specific rate of tympanostomy tube insertion across years created from age-period contingency table. Next, median polish analyses for log-transforming rates were performed. The residuals generated from the previous step were used to estimate the relative magnitude of cohort effects using linear regression. The exponentiated regression coefficients indicate the excess rate attributable to each cohort category. 
TABLE 1: The case number and rate of tympanostomy tube insertion in each age stratum and each calendar year.

\begin{tabular}{|c|c|c|c|c|c|c|c|c|c|c|c|c|c|c|c|c|c|c|c|c|}
\hline \multirow{2}{*}{ Age } & \multicolumn{2}{|c|}{2000} & \multicolumn{2}{|c|}{2001} & \multicolumn{2}{|c|}{2002} & \multicolumn{2}{|c|}{2003} & \multicolumn{2}{|c|}{2004} & \multicolumn{2}{|c|}{2005} & \multicolumn{2}{|c|}{2006} & \multicolumn{2}{|c|}{2007} & \multicolumn{2}{|c|}{2008} & \multicolumn{2}{|c|}{2009} \\
\hline & $n$ & Rate $^{\mathrm{a}}$ & $n$ & Rate $^{\mathrm{a}}$ & $n$ & Rate $^{\mathrm{a}}$ & $n$ & Rate $^{\mathrm{a}}$ & $n$ & Rate $^{\mathrm{a}}$ & $n$ & Rate $^{\mathrm{a}}$ & $n$ & Rate $^{\mathrm{a}}$ & $n$ & Rate $^{\mathrm{a}}$ & $n$ & Rate $^{\mathrm{a}}$ & $n$ & Rate $^{\mathrm{a}}$ \\
\hline Total & 1347 & 24.9 & 1406 & 26.5 & 1321 & 25.4 & 1109 & 21.6 & 1287 & 25.5 & 1389 & 28.3 & 1338 & 27.9 & 1253 & 26.8 & 1453 & 31.9 & 1171 & 26.5 \\
\hline & 106 & 36.2 & 143 & 58.0 & 116 & 49.0 & 140 & 64.4 & 121 & 58.5 & 71 & 36.3 & 86 & 44.6 & 76 & 39.6 & 115 & & 90 & 49.3 \\
\hline & 136 & 48.1 & 153 & 50.0 & 172 & 66.9 & 168 & 68.2 & 129 & 56.7 & 107 & 49.0 & 77 & 37.0 & 82 & 39.7 & 78 & 38.0 & 60 & 30.2 \\
\hline & 76 & 28.4 & 42 & 14.8 & 59 & 19.2 & 52 & 20.2 & 50 & 20.3 & 63 & 27.7 & 42 & 19.2 & 34 & 16.3 & 60 & 29.0 & 51 & 24.8 \\
\hline & 110 & 34.0 & 117 & & 80 & 28.3 & 59 & & 96 & & 117 & 47.5 & 98 & & 97 & & 94 & & 68 & 32.9 \\
\hline & 199 & 61.6 & 216 & 66.7 & 190 & 71.0 & 156 & 55.2 & 202 & 66.0 & 253 & 98.4 & 218 & 88.5 & 214 & 94.1 & 230 & 105.3 & 211 & 101.3 \\
\hline & 216 & 66.5 & 234 & 72.4 & 216 & 66.7 & 166 & 62.0 & 222 & 78.5 & 237 & 77.3 & 249 & 96.8 & 247 & 100.2 & 261 & 114.7 & 203 & 92.8 \\
\hline & 178 & 55.1 & 184 & 56.6 & 165 & & 119 & 36.8 & 163 & & 179 & 63.2 & 174 & 56.8 & 181 & 70.4 & 200 & 31.1 & 152 & 66.7 \\
\hline 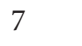 & 98 & 30.0 & 95 & 29.4 & 99 & 30.5 & 77 & 23.9 & 79 & 24.4 & 95 & 35.5 & 98 & 34.6 & 80 & 26.1 & 110 & 42.8 & 97 & 39.4 \\
\hline 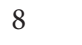 & 62 & 19.3 & 59 & 18.0 & 64 & 19.8 & 40 & 12.3 & 63 & 19.5 & 81 & 25.0 & 71 & 26.6 & 66 & 23.3 & 71 & 23.2 & 64 & 24.9 \\
\hline & 44 & 13.8 & 47 & 14.6 & 45 & 13.8 & 45 & 14.0 & 42 & 12.9 & 59 & 18.3 & 65 & 0.1 & 43 & & 69 & 24.4 & 44 & 14.4 \\
\hline 10 & 32 & 9.5 & 44 & 13.8 & 22 & 6.9 & 27 & & 40 & 12.4 & 30 & & 47 & 14.6 & 44 & & 49 & 18.3 & 35 & 12.4 \\
\hline & 37 & 11.9 & 23 & 6.9 & 36 & 11.3 & 16 & 5.0 & 24 & 7.4 & 41 & 12.7 & 48 & 14.8 & 34 & 10.5 & 49 & 15.2 & 22 & 8.2 \\
\hline 12 & 21 & & 25 & 8.0 & 27 & 8.1 & 14 & & 16 & 5. & 25 & 7.7 & 33 & 10.2 & 12 & & 26 & 8.1 & 37 & 11.4 \\
\hline 10 & 17 & 5.5 & 8 & 2.3 & 11 & 3.5 & 12 & 3.6 & 16 & 5.0 & 13 & 4.1 & 17 & 5.2 & 15 & 4.7 & 15 & 4.6 & 14 & 4.3 \\
\hline 1 & 7 & 2.3 & 8 & 2.6 & 7 & 2.1 & 12 & 3.9 & 10 & 3.0 & 9 & 2.8 & 4 & 1.2 & 14 & 4.3 & 12 & 3.7 & 11 & 3.4 \\
\hline 10 & 3 & 0.9 & 5 & 1.7 & 7 & 2.3 & 3 & 0.9 & 11 & 3.5 & 4 & 1.2 & 6 & 1.9 & 6 & 1.9 & 9 & 2.8 & 4 & 1.2 \\
\hline 10 & 5 & 1.4 & 3 & 0.9 & 5 & 1.7 & 3 & 1.0 & 3 & 0.9 & 5 & 1.6 & 5 & 1.5 & 8 & 2.5 & 5 & 1.6 & 8 & 2.5 \\
\hline
\end{tabular}

${ }^{\mathrm{a}}$ Incidence rate per 100,000 .

TABLE 2: Estimates of piecewise terms in Poisson regression models for evaluation of effects of pneumococcal conjugate vaccines against pneumococcal infection on tympanostomy tube insertion among children.

\begin{tabular}{lcccccrrr}
\hline & \multicolumn{2}{c}{$0-2$ years old } & \multicolumn{2}{c}{$2-5$ years old } & \multicolumn{2}{c}{ 5-9 years old } & \multicolumn{2}{c}{ 9-17 years old } \\
& $\beta$ & se & $\beta$ & se & $\beta$ & se & \multicolumn{1}{c}{$\beta$} \\
\hline Before 2005 & 0.004 & 0.014 & $0.052^{*}$ & 0.012 & $0.029^{*}$ & 0.009 & $0.037^{*}$ \\
After 2005 & $-0.074^{*}$ & 0.021 & $0.041^{*}$ & 0.015 & $0.064^{*}$ & 0.012 & 0.017 \\
${ }^{*} P<0.05$. & & & & & & & 0.020 \\
\hline
\end{tabular}

TABLE 3: Estimated rate ratios and $95 \%$ confidence intervals for the effect of birth cohort on tympanostomy tube insertion rate per population of 100,000 .

\begin{tabular}{lcc}
\hline & RR & $95 \%$ CI \\
\hline $2002-2003$ cohort & 1.00 & \\
$2004-2005$ cohort & 0.90 & $0.83-0.97^{*}$ \\
$2006-2007$ cohort & 0.21 & $0.19-0.23^{*}$ \\
\hline
\end{tabular}

RR: rate ratios.

CI: confidence interval.

\section{Results}

From the year 2000 to $2009,13,074$ patients receiving tympanostomy tube insertion under 17 years of age were included into this study. The patient numbers and tympanostomy tube insertion rates in each age stratum and in each calendar year were showed in Table 1. The tube insertion rates for children under 17 years of age ranged from 21.6 to 31.9 for 100,000 persons/year. The tympanostomy tube insertion rate of children showed an overall increasing trend with time except for children under 2 years old, which had a decreasing trend after 2005. There was a surge of increased rates in 2008 for children under 9 years old and then a decrease in 2009, which may have been the effect of the increased availability of imported vaccine in 2008 (Figure 1). The tympanostomy tube insertion rate for children less than 2 years old decreased significantly after $2005(\beta=-0.074, P<0.05$, and the negative $\beta$ value means a downward trend) and increased in children 2 to 9 years of age throughout the study period (positive $\beta$ values which mean upward trends, $P<0.05$ ) (Table 2). However, the tympanostomy tube insertion rate for children less than 2 years old seemed to be decreasing since 2003 before the introduction of the PCV7 in 2005 (Figure 1). The tympanostomy tube insertion rates of birth cohorts 20042005 and 2006-2007 were significantly lower than that of the birth cohort $2002-2003$ ( $R R=0.90$ and $0.21,95 \%$ CI $0.83-0.97$ and $0.19=0.23$, resp.). The risk of tube insertion of the birth cohort 2006-2007 after the introduction of the PCV7 was only one-fifth that of the birth cohort 2002-2003 before the introduction of the vaccine in Taiwan (Figure 2 and Table 3 ).

\section{Discussion}

Otitis media is a very common and important otologic problem in children. This condition is one of the most 


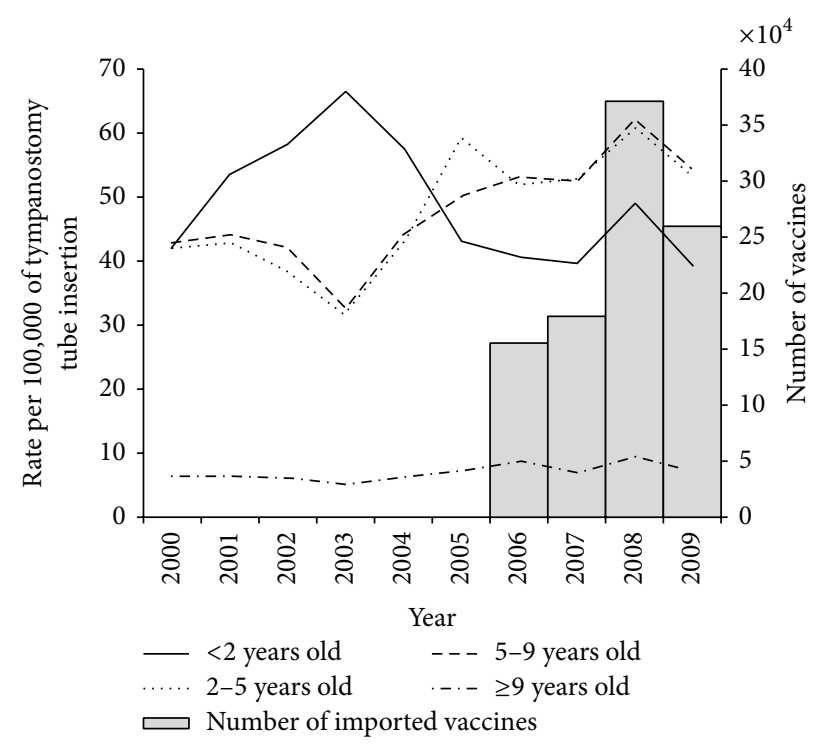

FIgURE 1: Age-specific rate of tympanostomy tube insertion from 2000 to 2009 and number of imported pneumococcal conjugate vaccines from 2006 to 2009.

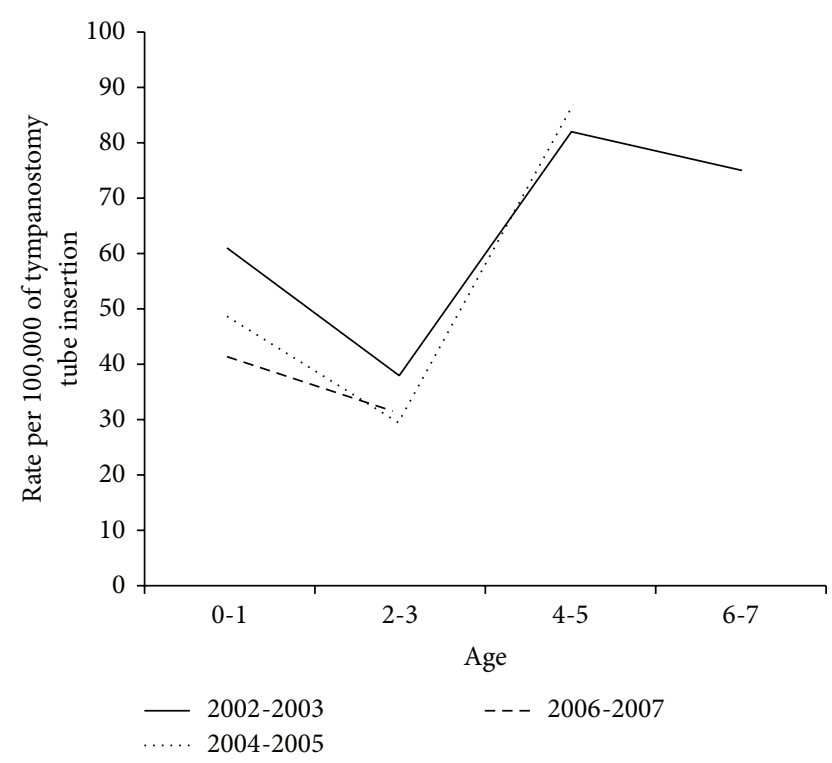

FIGURE 2: Birth cohort-specific rate of tympanostomy tube insertion across ages.

frequent causes of clinic visits and antibiotics prescriptions in children [36-38]. The surgical management of complicated and intractable otitis media, involving tympanostomy tube insertions, is one of the most frequent surgical procedures for children requiring general anesthesia [63]. Thus the prevention of pediatric otitis media is a very important issue. The most common pathogen of pediatric otitis media is Streptococcus pneumonia, which accounts for $30 \%$ to $50 \%$ of the pathogens associated with pediatric acute otitis media [2]. PCV7 can only offer partial protection against pediatric otitis media. We can see the protective effect of PCV7 on tube insertion in this study which meant the magnitude of reduction on tube insertion caused by pneumococcus would be even more significant. PCV7 was not initially included into national immunization program in Taiwan. According to the data from Taiwan Center of Disease Control, there were 962707 doses of PCV7 imported to Taiwan by the end of the year 2009. There were 798916 babies born in Taiwan from 2006 to 2009 and there were 1956143 children under 9 years of age in Taiwan in 2009 [60]. Newborn infants after 2005 could receive 1 to 4 doses of the vaccine and children who were 2-9 years old after 2006 could receive 1 dose of the vaccine. It is estimated that there could be up to $30.13 \%$ newborn infants after 2005 who were vaccinated or at most $49.2 \%$ of children under 9 years old who were immunized in 2009. To the best of our knowledge, this study is the first that has attempted to uncover the protective effect of PCV7 on pediatric otitis media or tympanostomy tube insertion in a partial vaccinated pediatric population. In this study, we found the decreased tympanostomy tube insertion rate in children younger than 2 years of age after the introduction of PCV7 in 2005 by period effects; however we also found that the decreased tympanostomy tube rate seemed to be started in 2003. As a result we could not conclude that the decreased tympanostomy tube rate was the protective effect Of PCV7. For children over 2 years of age, the tympanostomy tube insertion rate increased through the study period from 2000 to 2009 which meant we could not see the protective effect of PCV7 on tympanostomy tube insertions for children older than 2 years of age. However, we also found the tympanostomy tube insertion rate decreased significantly to one-fifth in the birth cohort (2006-2007 birth cohort) after introduction of the vaccine in comparison with the birth cohort (2002-2003 birth cohort) before the vaccine's introduction. This implied that there was a protective effect of PCV7 on tympanostomy tube insertion rates in this partially vaccinated population in Taiwan. In the analysis of cohort effect, we found children of 2004-2005 and 2006-2007 birth cohort had lower rates of tympanostomy tube insertion than that of children of 2002-2003 birth cohort before the age of 3 . The difference of rates of tympanostomy tube insertion in children between 2002-2003 and 2004-2005 birth cohorts disappeared when the age reached 4 to 5 . (Figure 2) The reason of these results we found may be that no PCV7 was available in Taiwan when children of 2002-2003 were younger than 3 years old and none of them was vaccinated. When children of 2002-2003 birth cohort reached 4 years old, PCV7 was introduced to Taiwan and some of them were vaccinated and became immunized leading to similar tympanostomy tube insertion rate with children of 20042005 birth cohort. This further supported the protective effect of PCV7 on tympanostomy tube insertion in this partial immunized pediatric population in Taiwan.

In this study, we found an overall increased trend of tympanostomy tube insertion rates in the study period from 2000 to 2009 in Taiwan (except for children under 2 years old after the introduction of PCV7 in 2005). Other studies have reported a downward trend of tympanostomy tube insertion in the United States from 1995 to 2005 [37, 47, 49, 50, 52, 59]. The increased trend in tube insertion rates in Taiwan may have been due to the progressive improvement accessibility 
of medical service since the beginning of National Health Insurance in 1995. Other factors such as better training of clinicians and diagnostic instruments have continuously been improving in Taiwan over the past decade. One other possible explanation of increased trend of tube insertion even after introduction of PCV7 is serotype replacement of pneumococcus. According to the report of Taiwan Centers for Disease Control, IPD in children under 5 years old caused by serotype $19 \mathrm{~A}$ increased from $12.2 \%$ in 2008 to $43.4 \%$ in 2011 . The increase of this multidrug resistant strain may also lead to the increase of intractable pediatric otitis media. Despite the increasing trend of tympanostomy tube insertion in Taiwan, we have a lower rate of tympanostomy tube insertion in comparison with that in the United States and Europe [6466]. The reasons may be that surgical interventions are not favorable choices of Taiwanese parents, leading to more conservative management, or otolaryngologists in Taiwan adhered more to the suggestions of clinical practice guideline in comparison to surgeons in the United States on treatment of pediatric otitis media with effusion [67-69]. For previous studies analyzing observational database, several of them also found protective effect PCV on otitis media for children $[9,37,47,48]$. Poehling et al. demonstrated less frequent otitis media visits and tube insertions in a more recent cohort of children covered by Tenn Care and New York private insurance [48]. However, we found those similar protective effects in a partially immunized pediatric population without a universal immunization program for PCV7.

To improve the internal validity of this study, tympanostomy tube insertion is used instead of diagnosis codes in ICD9 as a surrogate of chronic OME and recurrent AOM for the accuracy of defining the study population. If there was a code for certain surgical procedures for a particular patient in the claims data, that patient was considered to have the disease and therefore underwent a surgical procedure to address it. In contrast, if diagnosis codes in ICD-9 were used as a surrogate for the disease, the probability of miscoding by the physician could have been much higher. Physicians may use the wrong diagnosis code during instances of misdiagnoses. They also may do this for prescribing antibiotics or laboratory test in order to pass the review of the insurance payer or to improve reimbursement.

In this study we used a population-based database, NHIRD, without sampling for the analysis. However, the major limitation of this study is the limitation of the administrative claims data. Medical records and the operative notes of every patient could not be obtained. Another limitation is that, at the beginning of PCV7 vaccination in Taiwan, PCV7 was only for select patients. PCV was not included in national universal vaccination program until 2013. We could not identify those vaccinated children in the NHIRD in Taiwan. Therefore, we were unable to examine the difference of tympanostomy insertion rates between vaccinated and unvaccinated children, which means we could not determine if there was a direct effect of PCV7 on tympanostomy tube insertion rates. For this reason, we could not distinguish whether the protective effect was due to a direct effect or indirect (herd) effect. We can only make the inference that the protective effect was a direct effect or combination of both direct and indirect effects. There are other limitations of this study. There is surveillance bias in the study. Parents who are willing to pay for the vaccine may care more about the health condition of the children and may seek for medical care more. The vaccinated children may be easier to be picked up to have middle ear condition than unvaccinated children. Fortunately, this surveillance bias leads to underestimation of the protective effect of the vaccine which may not change the direction of the result of this study. In this study we use the imported vaccine number as the surrogate of total vaccine delivered to the children. This must be an overestimation of the vaccination which also leads to underestimation of the protective effect of the vaccine.

\section{Conclusion}

Tympanostomy tube insertion rates in birth cohorts after 2005 were lower than those in the birth cohort before 2005 . PCV7 may have protective effect on pediatric tympanostomy tube insertion rates in a partially immunized population in Taiwan.

$\begin{array}{ll}\text { Abbreviations } \\ \text { AAO-HNS: } \\ & \begin{array}{l}\text { American Academy of } \\ \text { Otolaryngology-Head and Neck } \\ \text { Surgery }\end{array} \\ & \text { Acute otitis media } \\ \text { AOM: } & \text { Confidence interval } \\ \text { CI: } & \text { Invasive pneumococcal disease } \\ \text { IPD: } & \text { National Health Insurance Research } \\ \text { NHIRD: } & \text { Database } \\ \text { PCV7: } & \text { Seven-valent pneumococcal conjugate } \\ & \text { vaccine } \\ \text { OME: } & \text { Otitis media with effusion } \\ \text { RR: } & \text { Rate ratio. }\end{array}$

\section{Disclosure}

The funders had no role in study design, data collection and analysis, decision to publish, or preparation of the paper.

\section{Conflict of Interests}

All authors have no conflict of interests to disclose.

\section{Acknowledgments}

This study was funded by research grant of Taipei Veterans General Hospital (V103B-035) and Taipei Veterans General Hospital, National Yang-Ming University, Excellent Physician Scientists Cultivation Program, no. 103-V-B-054.

\section{References}

[1] M. M. Rovers, A. G. M. Schilder, G. A. Zielhuis, and R. M. Rosenfeld, "Otitis media," The Lancet, vol. 363, no. 9407, pp. 465-473, 2004.

[2] J. O. Hendley, "Otitis media," The New England Journal of Medicine, vol. 347, no. 15, pp. 1169-1174, 2002. 
[3] M. Tos, "Epidemiology and natural history of secretory otitis," The American Journal of Otology, vol. 5, no. 6, pp. 459-462, 1984.

[4] J. L. Paradise, H. E. Rockette, D. K. Colborn et al., "Otitis media in 2253 Pittsburgh-area infants: prevalence and risk factors during the first two years of life," Pediatrics, vol. 99, no. 3, pp. 318-333, 1997.

[5] S. E. Stool, A. O. Berg, S. Berman et al., Otitis Media with Effusion in Young Children. Clinical Practice Guideline, AHCPR Publication No. 94-0622, number 12, Agency for Health Care Policy and Research, Public Health Service, US Department of Health and Human Services, Rockville, Md, USA, 1994.

[6] I. G. Williamson, J. Dunleavey, J. Bain, and D. Robinson, “The natural history of otitis media with effusion-a three-year study of the incidence and prevalence of abnormal tympanograms in four South West Hampshire Infant and First schools," The Journal of Laryngology \& Otology, vol. 108, no. 11, pp. 930-934, 1994.

[7] R. M. Rosenfeld, L. Culpepper, K. J. Doyle et al., "Clinical practice guideline: otitis media with effusion," OtolaryngologyHead and Neck Surgery, vol. 130, no. 5, supplement, pp. S95S118, 2004.

[8] R. M. Rosenfeld, S. R. Schwartz, M. A. Pynnonen et al., "Clinical practice guideline: tympanostomy tubes in children," Otolaryngology: Head and Neck Surgery, vol. 149, no. 1, supplement, pp. S1-S35, 2013.

[9] K. A. Poehling, B. J. Lafleur, P. G. Szilagyi et al., "Populationbased impact of pneumococcal conjugate vaccine in young children," Pediatrics, vol. 114, no. 3, pp. 755-761, 2004.

[10] D. M. Musher, "Pneumococcal vaccine-direct and indirect ('herd') effects," The New England Journal of Medicine, vol. 354, no. 14, pp. 1522-1524, 2006.

[11] A. Bechini, S. Boccalini, and P. Bonanni, "Immunization with the 7-valent conjugate pneumococcal vaccine: Impact evaluation, continuing surveillance and future perspectives," Vaccine, vol. 27, no. 25-26, pp. 3285-3290, 2009.

[12] T. Pilishvili, C. Lexau, M. M. Farley et al., "Sustained reductions in invasive pneumococcal disease in the era of conjugate vaccine," The Journal of Infectious Diseases, vol. 201, no. 1, pp. 32-41, 2010.

[13] F. Ansaldi, D. de Florentis, P. Canepa et al., "Epidemiological changes after PCV7 implementation in Italy," Human Vaccines, vol. 7, pp. S211-S216, 2011.

[14] S. Ben-Shimol, D. Greenberg, N. Givon-Lavi et al., "Rapid reduction in invasive pneumococcal disease after introduction of PCV7 into the National Immunization Plan in Israel," Vaccine, vol. 30, no. 46, pp. 6600-6607, 2012.

[15] M. A. Rose, D. Christopoulou, T. T. H. Myint, and I. de Schutter, "The burden of invasive pneumococcal disease in children with underlying risk factors in North America and Europe," International Journal of Clinical Practice, vol. 68, no. 1, pp. 8-19, 2014.

[16] I. N. Grivea, M. Panagiotou, A. G. Tsantouli, and G. A. Syrogiannopoulos, "Impact of heptavalent pneumococcal conjugate vaccine on nasopharyngeal carriage of penicillin-resistant streptococcus pneumoniae among day-care center attendees in Central Greece," The Pediatric Infectious Disease Journal, vol. 27, no. 6, pp. 519-525, 2008.

[17] A. Fenoll, L. Aguilar, J. J. Granizo et al., "Has the licensing of respiratory quinolones for adults and the 7-valent pneumococcal conjugate vaccine (PCV-7) for children had herd effects with respect to antimicrobial non-susceptibility in invasive Streptococcus pneumoniae?" Journal of Antimicrobial Chemotherapy, vol. 62, no. 6, pp. 1430-1433, 2008.

[18] R. Dagan and K. P. Klugman, "Impact of conjugate pneumococcal vaccines on antibiotic resistance," The Lancet Infectious Diseases, vol. 8, no. 12, pp. 785-795, 2008.

[19] S. S. Richter, K. P. Heilmann, C. L. Dohrn, F. Riahi, S. E. Beekmann, and G. V. Doern, "Changing epidemiology of antimicrobial-resistant Streptococcus pneumoniae in the United States, 2004-2005," Clinical Infectious Diseases, vol. 48, no. 3, pp. e23-e33, 2009.

[20] S. Guevara, A. Abdelnour, C. Soley, N. Porat, R. Dagan, and A. Arguedas, "Streptococcus pneumoniae serotypes isolated from the middle ear fluid of Costa Rican children following introduction of the heptavalent pneumococcal conjugate vaccine into a limited population," Vaccine, vol. 30, no. 26, pp. 3857-3861, 2012.

[21] J.-H. Song, "Advances in pneumococcal antibiotic resistance," Expert Review of Respiratory Medicine, vol. 7, no. 5, pp. 491-498, 2013.

[22] A. Roca, C. Bottomley, P. C. Hill et al., "Effect of age and vaccination with a pneumococcal conjugate vaccine on the density of pneumococcal nasopharyngeal carriage," Clinical Infectious Diseases, vol. 55, no. 6, pp. 816-824, 2012.

[23] N. H. T. M. Dukers-Muijrers, E. Stobberingh, P. Beisser, R. C. H. Boesten, P. Jacobs, and C. J. P. A. Hoebe, "Nasal carriage of Streptococcus pneumoniae serotypes and Staphylococcus aureus in Streptococcus pneumoniae-vaccinated and non-vaccinated young children," Epidemiology and Infection, vol. 141, no. 3, pp. 631-638, 2013.

[24] P. C. Wroe, G. M. Lee, J. A. Finkelstein et al., "Pneumococcal carriage and antibiotic resistance in young children before 13-valent conjugate vaccine," The Pediatric Infectious Disease Journal, vol. 31, no. 3, pp. 249-254, 2012.

[25] J. Spijkerman, S. M. P. J. Prevaes, E. J. M. van Gils et al., "Longterm effects of pneumococcal conjugate vaccine on nasopharyngeal carriage of $S$. pneumoniae, $S$. aureus, $H$. influenzae and M. catarrhalis," PLoS ONE, vol. 7, no. 6, Article ID e39730, 2012.

[26] N. B. Halasa, C. G. Grijalva, P. G. Arbogast et al., "Nearly complete elimination of the 7-valent pneumococcal conjugate vaccine serotypes in Tennessee," Pediatric Infectious Disease Journal, vol. 32, no. 6, pp. 604-609, 2013.

[27] K. E. Fleming-Dutra, L. Conklin, J. D. Loo et al., "Systematic review of the effect of pneumococcal conjugate vaccine dosing schedules on vaccine-type nasopharyngeal carriage," The Pediatric Infectious Disease Journal, vol. 33, no. 2, supplement, pp. S152-S160, 2014.

[28] C. G. Whitney, M. M. Farley, J. Hadler et al., "Decline in invasive pneumococcal disease after the introduction of proteinpolysaccharide conjugate vaccine," The New England Journal of Medicine, vol. 348, no. 18, pp. 1737-1746, 2003.

[29] H. E. Hsu, K. A. Shutt, M. R. Moore et al., "Effect of pneumococcal conjugate vaccine on pneumococcal meningitis," The New England Journal of Medicine, vol. 360, no. 3, pp. 244-256, 2009.

[30] L. Simonsen, R. J. Taylor, Y. Young-Xu, M. Haber, L. May, and K. P. Klugman, "Impact of pneumococcal conjugate vaccination of infants on pneumonia and influenza hospitalization and mortality in all age groups in the United States," mBio, vol. 2, no. 1, Article ID e00309-10, 2011.

[31] B. A. Kendall, K. K. Dascomb, R. R. Mehta et al., "Streptococcus pneumoniae serotypes in Utah adults at the end of the PCV7 era," Vaccine, vol. 29, no. 49, pp. 9123-9126, 2011. 
[32] M. T. T. Htar, H. Madhava, P. Balmer, D. Christopoulou, D. Menegas, and E. Bonnet, "A review of the impact of pneumococcal polysaccharide conjugate vaccine (7-valent) on pneumococcal meningitis," Advances in Therapy, vol. 30, no. 8, pp. 748762, 2013.

[33] S. M. Davis, M. Deloria-Knoll, H. T. Kassa, and K. L. O’Brien, "Impact of pneumococcal conjugate vaccines on nasopharyngeal carriage and invasive disease among unvaccinated people: review of evidence on indirect effects," Vaccine, vol. 32, no. 1, pp. 133-145, 2013.

[34] L. Conklin, J. D. Loo, J. Kirk et al., "Systematic review of the effect of pneumococcal conjugate vaccine dosing schedules on vaccine-type invasive pneumococcal disease among young children," The Pediatric Infectious Disease Journal, vol. 33, no. 2, pp. S109-S118, 2014.

[35] J. D. Grabenstein and D. J. Weber, "Pneumococcal serotype diversity among adults in various countries, influenced by pediatric pneumococcal vaccination uptake," Clinical Infectious Diseases, vol. 58, no. 6, pp. 854-864, 2014.

[36] G. D. Overturf, “Technical report: prevention of pneumococcal infections, including the use of pneumococcal conjugate and polysaccharide vaccines and antibiotic prophylaxis," Pediatrics, vol. 106, no. 2, part 1, pp. 367-376, 2000.

[37] F. Zhou, A. Shefer, Y. Kong, and J. P. Nuorti, "Trends in acute otitis media-related health care utilization by privately insured young children in the United States, 1997-2004," Pediatrics, vol. 121, no. 2, pp. 253-260, 2008.

[38] M. A. O’Brien, L. A. Prosser, J. L. Paradise et al., "New vaccines against otitis media: projected benefits and cost-effectiveness," Pediatrics, vol. 123, no. 6, pp. 1452-1463, 2009.

[39] S. Black, H. Shinefield, B. Fireman et al., "Efficacy, safety and immunogenicity of heptavalent pneumococcal conjugate vaccine in children. Northern California Kaiser Permanente Vaccine Study Center Group," The Pediatric Infectious Disease Journal, vol. 19, no. 3, pp. 187-195, 2000.

[40] J. Eskola, T. Kilpi, A. Palmu et al., "Efficacy of a pneumococcal conjugate vaccine against acute otitis media," The New England Journal of Medicine, vol. 344, no. 6, pp. 403-409, 2001.

[41] S. Black and H. Shinefield, "Safety and efficacy of the sevenvalent pneumococcal conjugate vaccine: evidence from Northern California," European Journal of Pediatrics, Supplement, vol. 161, no. 2, supplement, pp. S127-S131, 2002.

[42] B. Fireman, S. B. Black, H. R. Shinefield, J. Lee, E. Lewis, and P. Ray, "Impact of the pneumococcal conjugate vaccine on otitis media," The Pediatric Infectious Disease Journal, vol. 22, no. 1, pp. 10-16, 2003.

[43] A. A. I. Palmu, J. Verho, J. Jokinen, P. Karma, and T. M. Kilpi, "The seven-valent pneumococcal conjugate vaccine reduces tympanostomy tube placement in children," The Pediatric Infectious Disease Journal, vol. 23, no. 8, pp. 732-738, 2004.

[44] T. Kilpi, H. Åhman, J. Jokinen et al., "Protective efficacy of a second pneumococcal conjugate vaccine against pneumococcal acute otitis media in infants and children: randomized, controlled trial of a 7-valent pneumococcal polysaccharidemeningococcal outer membrane protein complex conjugate vaccine in 1666 children," Clinical Infectious Diseases, vol. 37, no. 9, pp. 1155-1164, 2003.

[45] R. Prymula, P. Peeters, V. Chrobok et al., "Pneumococcal capsular polysaccharides conjugated to protein $\mathrm{D}$ for prevention of acute otitis media caused by both Streptococcus pneumoniae and non-typable Haemophilus influenzae: a randomised doubleblind efficacy study," The Lancet, vol. 367, no. 9512, pp. 740-748, 2006.

[46] K. L. O’Brien, A. B. David, A. Chandran et al., "Randomized, controlled trial efficacy of pneumococcal conjugate vaccine against otitis media among navajo and white mountain apache infants," The Pediatric Infectious Disease Journal, vol. 27, no. 1, pp. 71-73, 2008.

[47] C. G. Grijalva, K. A. Poehling, J. P. Nuorti et al., "National impact of universal childhood immunization with pneumococcal conjugate vaccine on outpatient medical care visits in the United States," Pediatrics, vol. 118, no. 3, pp. 865-873, 2006.

[48] K. A. Poehling, P. G. Szilagyi, C. G. Grijalva et al., "Reduction of frequent otitis media and pressure-equalizing tube insertions in children after introduction of pneumococcal conjugate vaccine," Pediatrics, vol. 119, no. 4, pp. 707-715, 2007.

[49] C. M. Sox, J. A. Finkelstein, R. Yin, K. Kleinman, and T. A. Lieu, "Trends in otitis media treatment failure and relapse," Pediatrics, vol. 121, no. 4, pp. 674-679, 2008.

[50] C. G. Grijalva, J. P. Nuorti, and M. R. Griffin, “Antibiotic prescription rates for acute respiratory tract infections in US ambulatory settings," The Journal of the American Medical Association, vol. 302, no. 7, pp. 758-766, 2009.

[51] P. D. Wals, M. Carbon, E. Sévin, G. Deceuninck, and M. Ouakki, "Reduced physician claims for otitis media after implementation of pneumococcal conjugate vaccine program in the province of Quebec, Canada," The Pediatric Infectious Disease Journal, vol. 28, no. 9, pp. e271-e274, 2009.

[52] R. J. Singleton, R. C. Holman, R. Plant et al., "Trends in otitis media and myringtomy with tube placement among American Indian/Alaska native children and the US general population of children," The Pediatric Infectious Disease Journal, vol. 28, no. 2, pp. 102-107, 2009.

[53] T. Marom, A. Tan, G. S. Wilkinson, K. S. Pierson, J. L. Freeman, and T. Chonmaitree, "Trends in otitis media-related health care use in the united states, 2001-2011," JAMA Pediatrics, vol. 168, no. 1, pp. 68-75, 2014.

[54] S. I. Pelton, "Acute otitis media in the era of effective pneumococcal conjugate vaccine: will new pathogens emerge?" Vaccine, vol. 19, supplement 1, pp. S96-S99, 2000.

[55] C. J. Harrison, "Changes in treatment strategies for acute otitis media after full implementation of the pneumococcal seven valent conjugate vaccine," The Pediatric Infectious Disease Journal, vol. 22, no. 8, pp. S120-S130, 2003.

[56] R. Dagan, "The potential effect of widespread use of pneumococcal conjugate vaccines on the practice of pediatric otolaryngology: the case of acute otitis media," Current Opinion in Otolaryngology \& Head and Neck Surgery, vol. 12, no. 6, pp. 488494, 2004.

[57] S. Brunton, "Current face of acute otitis media: microbiology and prevalence resulting from widespread use of heptavalent pneumococcal conjugate vaccine," Clinical Therapeutics, vol. 28, no. 1, pp. 118-123, 2006.

[58] S. Taylor, P. Marchisio, A. Vergison, J. Harriague, W. P. Hausdorff, and M. Haggard, "Impact of pneumococcal conjugate vaccination on otitis media: a systematic review," Clinical Infectious Diseases, vol. 54, no. 12, pp. 1765-1773, 2012.

[59] S. P. Fitzwater, A. Chandran, M. Santosham, and H. L. Johnson, "The worldwide impact of the seven-valent pneumococcal conjugate vaccine," The Pediatric Infectious Disease Journal, vol. 31, no. 5, pp. 501-508, 2012. 
[60] Ministry of the Interior, "Taiwan National Statistics Report," December 2013, http://statis.moi.gov.tw/micst/stmain.jsp?sys= 100.

[61] A. K. Wagner, S. B. Soumerai, F. Zhang, and D. Ross-Degnan, "Segmented regression analysis of interrupted time series studies in medication use research," Journal of Clinical Pharmacy and Therapeutics, vol. 27, no. 4, pp. 299-309, 2002.

[62] K. M. Keyes and G. Li, "A multiphase method for estimating cohort effects in age-period contingency table data," Annals of Epidemiology, vol. 20, no. 10, pp. 779-785, 2010.

[63] R. Pokras, L. J. Kozak, and E. McCarthy, "Ambulatory and inpatient procedures in the United States, 1994," in Vital and Health Statatistics, Series 13, vol. 132, pp. 1-113, 1997.

[64] M.-C. Wang, Y.-P. Wang, C.-H. Chu, T.-Y. Tu, A.-S. Shiao, and P. Chou, "The protective effect of adenoidectomy on pediatric tympanostomy tube re-insertions: a population-based birth cohort study," PLoS ONE, vol. 9, no. 7, Article ID e101175, 2014.

[65] M. D. Kogan, M. D. Overpeck, H. J. Hoffman, and M. L. Casselbrant, "Factors associated with tympanostomy tube insertion among preschool-aged children in the United States," American Journal of Public Health, vol. 90, no. 2, pp. 245-250, 2000.

[66] K. J. Kværner, P. Nafstad, and J. J. K. Jaakkola, "Otolaryngological surgery and upper respiratory tract infections in children: an epidemiological study," Annals of Otology, Rhinology \& Laryngology, vol. 111, no. 11, pp. 1034-1039, 2002.

[67] M.-C. Wang, C.-K. Huang, Y.-P. Wang, and C.-W. Chien, "Effects of increased payment for ventilation tube insertion on decision making for paediatric otitis media with effusion," Journal of Evaluation in Clinical Practice, vol. 18, no. 4, pp. 919922, 2012.

[68] S. Keyhani, L. C. Kleinman, M. Rothschild et al., "Clinical characteristics of New York City children who received tympanostomy tubes in 2002," Pediatrics, vol. 121, no. 1, pp. e24-e33, 2008.

[69] S. Keyhani, L. C. Kleinman, M. Rothschild, J. M. Bernstein, R. Anderson, and M. Chassin, "Overuse of tympanostomy tubes in New York metropolitan area: evidence from five hospital cohort," British Medical Journal, vol. 337, article a1607, 2008. 


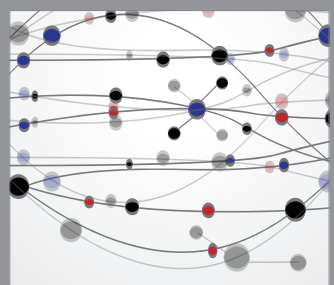

The Scientific World Journal
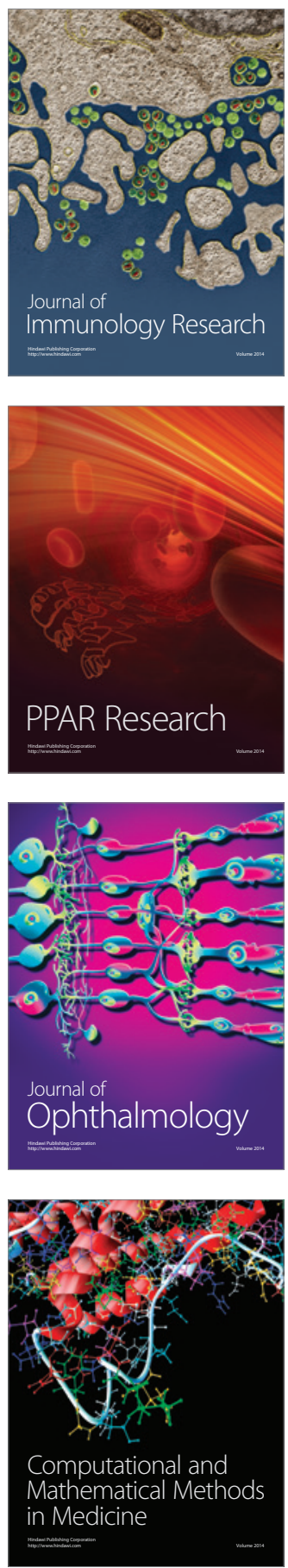

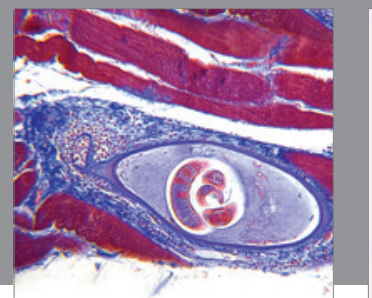

Gastroenterology

Research and Practice
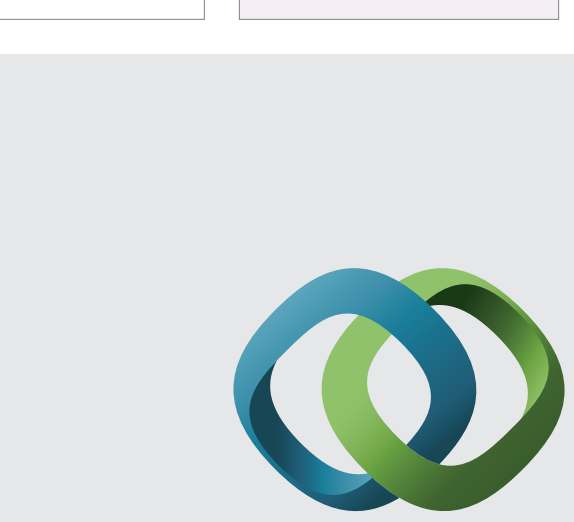

\section{Hindawi}

Submit your manuscripts at

http://www.hindawi.com
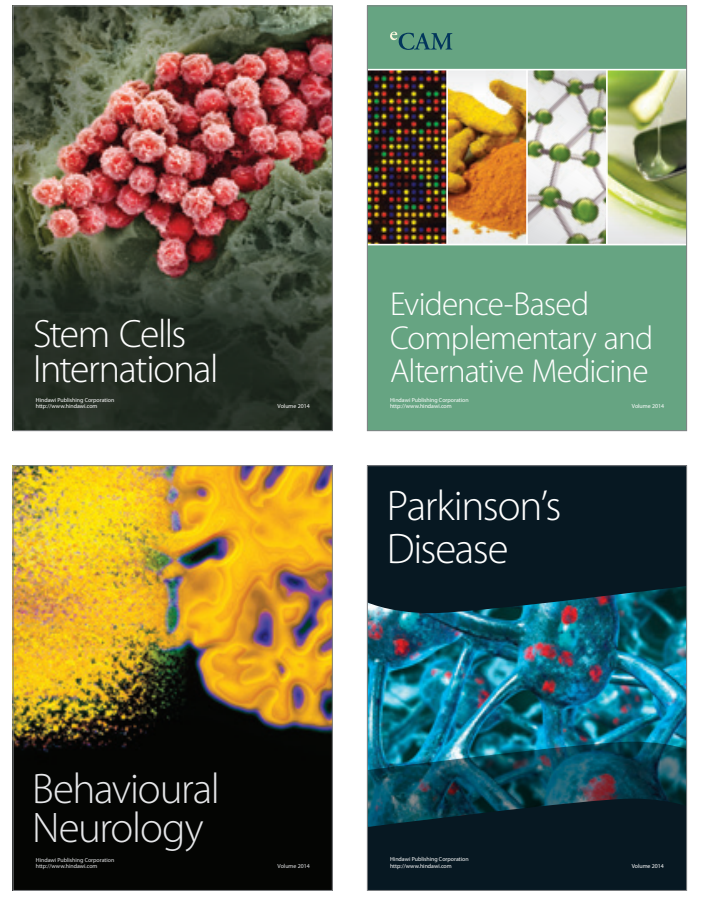
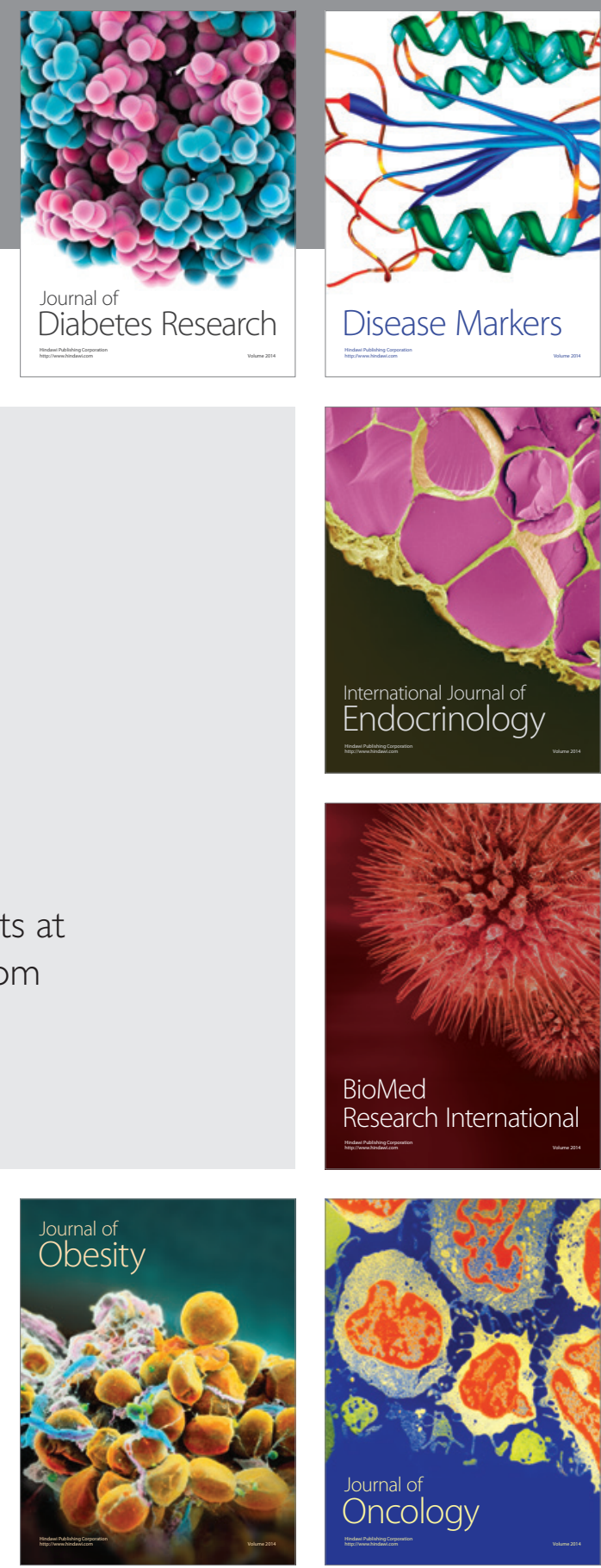

Disease Markers
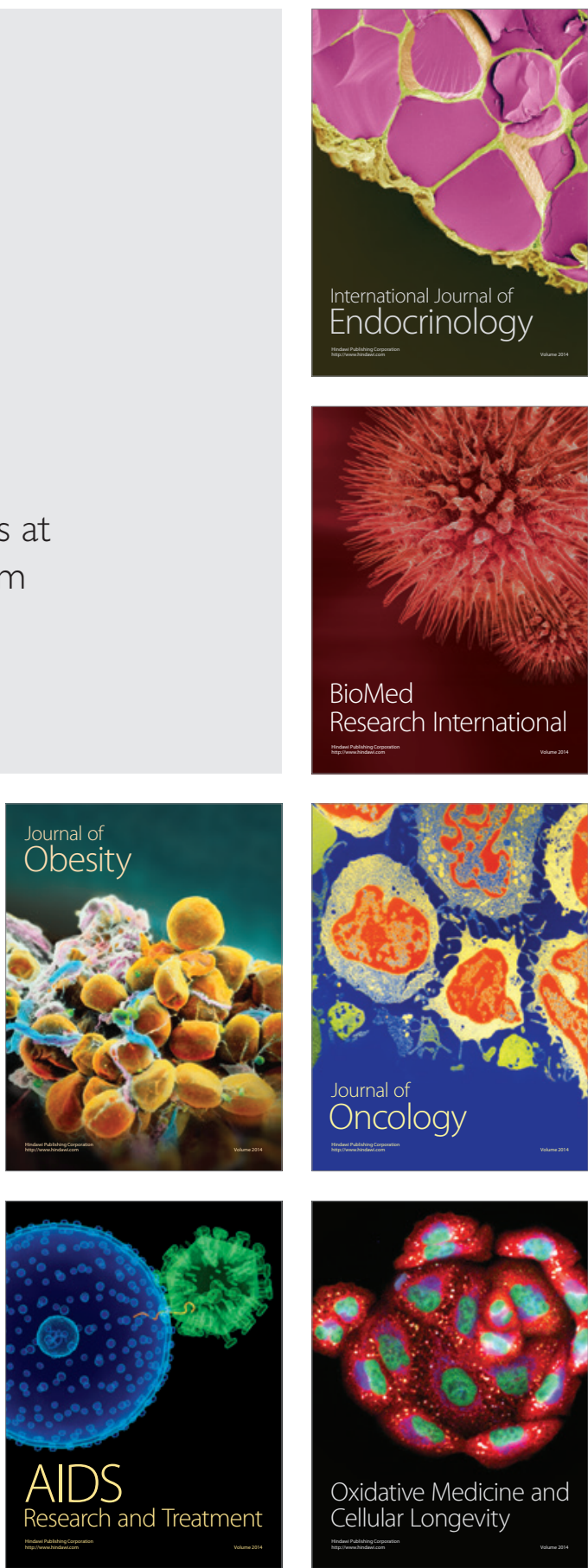